\title{
Synthesis of Decentralized Variable Gain Robust Controllers for Large-Scale Interconnected Systems with Structured Uncertainties
}

\author{
Shunya Nagai ${ }^{1}$ and Hidetoshi Oya ${ }^{2}$ \\ ${ }^{1}$ The Graduate School of Advanced Technology and Science, The University of Tokushima, 2-1 Minamijosanjima, \\ Tokushima 770-8506, Japan \\ ${ }^{2}$ The Institute of Technology and Science, The University of Tokushima, 2-1 Minamijosanjima, Tokushima 770-8506, Japan \\ Correspondence should be addressed to Shunya Nagai; s-nagai@ee.tokushima-u.ac.jp
}

Received 13 February 2014; Revised 7 May 2014; Accepted 8 May 2014; Published 9 July 2014

Academic Editor: Petko Petkov

Copyright (C) 2014 S. Nagai and H. Oya. This is an open access article distributed under the Creative Commons Attribution License, which permits unrestricted use, distribution, and reproduction in any medium, provided the original work is properly cited.

In this paper, we propose a decentralized variable gain robust controller which achieves not only robust stability but also satisfactory transient behavior for a class of uncertain large-scale interconnected systems. For the uncertain large-scale interconnected system, the uncertainties and the interactions satisfy the matching condition. The proposed decentralized robust controller consists of a fixed feedback gain controller and a variable gain one determined by a parameter adjustment law. In this paper, we show that sufficient conditions for the existence of the proposed decentralized variable gain robust controller are given in terms of LMIs. Finally, a simple numerical example is included.

\section{Introduction}

To design control systems, it is necessary to derive a mathematical model for controlled systems. However, there always exist some gaps between the mathematical model and the controlled system; that is, uncertainties between actual systems and mathematical models are unavoidable. Hence for dynamical systems with uncertainties, so-called robust controller design methods have been well studied in the past thirty years, and there are a large number of studies for linear uncertain dynamical systems (e.g., see $[1,2]$ and references therein). In particular for uncertain linear systems, several quadratic stabilizing controllers and $\mathscr{H}^{\infty}$ one have been suggested (e.g., [3-5]), and a connection between $\mathscr{H}^{\infty}$ control and quadratic stabilization has also been established [6]. In addition, several design methods of variable gain controllers for uncertain continuous-time systems have been shown (e.g., [7-9]). These robust controllers are composed of a fixed gain controller and a variable gain one, and variable gain controllers are tuned by updating laws. Especially, in Oya and Hagino [8] the error signal between the desired trajectory and the actual response is introduced and the variable gain controller is determined so as to reduce the effect of uncertainties.

On the other hand, the decentralized control for largescale interconnected systems has been widely studied, because large-scale interconnected systems can be seen in such diverse fields as economic systems, electrical systems, and so on. A large number of results in decentralized control systems can be seen in Šiljak [10]. A framework for the design of decentralized robust model reference adaptive control for interconnected time-delay systems has been considered in the work of Hua et al. [11] and decentralized fault tolerant control problem has also been studied [12]. Additionally, there are many existing results for decentralized robust control for large-scale interconnected systems with uncertainties (e.g., [13-16]). In Mao and Lin [13] for large-scale interconnected systems with unmodelled interaction, the aggregative derivation is tracked by using a model following technique with online improvement, and a sufficient condition for which the overall system when controlled by the completely decentralized control is asymptotically stable has been established. Gong [15] has proposed decentralized robust controllers which guarantee robust stability with prescribed degree of 
exponential convergence. Furthermore, some design methods of decentralized guaranteed cost controllers for uncertain large-scale interconnected systems have also been suggested $[17,18]$.

In this paper, on the basis of the existing results $[8,9]$, we propose a decentralized variable gain robust controller for a class of uncertain large-scale interconnected systems; that is, this study is an extension of the previous studies in this field. For the uncertain large-scale interconnected systems, uncertainties and interactions satisfy the matching condition. The proposed decentralized variable gain robust control input is defined as a state feedback with a fixed feedback gain matrix designed by using nominal subsystem and an error signal feedback with a fixed compensation gain matrix and a variable one determined by a parameter adjustment law. In this paper, LMI-based sufficient conditions for the existence of the proposed decentralized variable gain robust controller are derived. One can see that the crucial difference between the existing results and our new one is that the derived LMI conditions in this paper are always feasible; that is, the proposed decentralized variable gain robust control system can always be designed. Additionally, the number of LMIs needed to be solved equals the number of subsystems and is less than the conventional decentralized robust control with a fixed feedback gain matrix based on Lyapunov stability criterion. Therefore, the proposed decentralized robust control scheme is very useful.

This paper is organized as follows. We show notations and useful lemmas which are used in this paper in Section 2, and in Section 3, the class of uncertain large-scale interconnected systems under consideration is presented, and uncertain error subsystems and a decentralized variable gain robust control input are introduced. Section 4 contains the main results; that is, LMI-based sufficient conditions for the existence of the proposed decentralized variable gain robust controller are derived. Finally, simple illustrative examples are included to show the effectiveness of the proposed decentralized variable gain robust control system.

\section{Preliminaries}

In this section, notations and useful and well-known lemmas (see $[19,20]$ for details) which are used in this paper are shown.

In the paper, the following notations are used. For a matrix $\mathscr{X}$, the inverse of matrix $\mathscr{X}$ and the transpose of one are denoted by $\mathscr{X}^{-1}$ and $\mathscr{X}^{T}$, respectively. Additionally $H_{e}\{\mathscr{X}\}$ and $I_{n}$ mean $\mathscr{X}+\mathscr{X}^{T}$ and $n$-dimensional identity matrix, respectively, and the notation $\operatorname{diag}\left(\mathscr{X}_{1}, \ldots, \mathscr{X}_{\mathscr{M}}\right)$ represents a block diagonal matrix composed of matrices $\mathscr{X}_{i}$ for $i=$ $1, \ldots, \mathscr{M}$. For real symmetric matrices $\mathscr{X}$ and $\mathscr{Y}, \mathscr{X}>$ $\mathscr{Y}$ (resp., $\mathscr{X} \geq \mathscr{Y}$ ) means that $\mathscr{X}-\mathscr{Y}$ is positive (resp., nonnegative) definite matrix. For a vector $\alpha \in \mathbb{R}^{n},\|\alpha\|$ denotes standard Euclidian norm and,for a matrix $\mathscr{X},\|\mathscr{X}\|$ represents its induced norm. The symbols "ڤ” and “ $\star$ ” mean equality by definition and symmetric blocks in matrix inequalities, respectively.
Lemma 1. For arbitrary vectors $\lambda$ and $\xi$ and the matrices $\mathscr{G}$ and $\mathscr{H}$ which have appropriate dimensions, the following inequality holds:

$$
2 \lambda^{T} \mathscr{G} \Delta(t) \mathscr{H} \xi \leq 2\left\|\mathscr{G}^{T} \lambda\right\|\|\mathscr{H} \xi\|,
$$

where $\Delta(t)$ with appropriate dimensions is a time-varying unknown matrix satisfying $\|\Delta(t)\| \leq 1.0$.

Lemma 2 (Schur complement). For a given constant real symmetric matrix $\Xi$, the following items are equivalent:
(i) $\Xi=\left(\begin{array}{ll}\Xi_{11} & \Xi_{12} \\ \Xi_{12}^{T} & \Xi_{22}\end{array}\right)>0$,
(ii) $\Xi_{11}>0$ and $\Xi_{22}-\Xi_{12}^{T} \Xi_{11}^{-1} \Xi_{12}>0$,
(iii) $\Xi_{22}>0$ and $\Xi_{11}-\Xi_{12} \Xi_{22}^{-1} \Xi_{12}^{T}>0$.

\section{Problem Formulation}

Consider the uncertain large-scale interconnected system composed of $\mathcal{N}$ subsystems described as

$$
\frac{d}{d t} x_{i}(t)=A_{i i}\left(\theta_{i i}\right) x_{i}(t)+\sum_{\substack{j=1 \\ j \neq i}}^{\mathcal{N}} A_{i j}\left(\theta_{i j}\right) x_{j}(t)+B_{i} u_{i}(t)
$$

where $x_{i}(t) \in \mathbb{R}^{n_{i}}$ and $u_{i}(t) \in \mathbb{R}^{m_{i}}(i=1, \ldots, \mathcal{N})$ are the vectors of the state and the control input for the $i$ th subsystem, respectively, and the vector $x(t)=\left(x_{1}^{T}(t), \ldots, x_{\mathcal{N}}^{T}(t)\right)^{T}$ is the state of the overall system. The matrices $A_{i i}(t)$ and $A_{i j}(t)$ in (2) are given by

$$
\begin{aligned}
& A_{i i}\left(\theta_{i i}\right)=A_{i i}+\sum_{k=1}^{p_{i i}} \theta_{i i}^{(k)}(t) B_{i} \mathscr{E}_{i i}^{(k)}, \\
& A_{i j}\left(\theta_{i j}\right)=B_{i} \mathscr{D}_{i j}+\sum_{k=1}^{p_{i j}} \theta_{i j}^{(k)}(t) B_{i} \mathscr{E}_{i j}^{(k)} ;
\end{aligned}
$$

that is, the uncertainties and the interaction terms satisfy the matching condition. In (2) and (3), the matrices $A_{i i} \in$ $\mathbb{R}^{n_{i} \times n_{i}}, A_{i j} \in \mathbb{R}^{n_{i} \times n_{j}}$, and $B_{i} \in \mathbb{R}^{n_{i} \times m_{i}}$ denote the nominal values of the system, and the matrices $\mathscr{D}_{i j}, \mathscr{E}_{i i}^{(k)}$, and $\mathscr{E}_{i j}^{(k)}$ with appropriate dimensions represent the structure of interactions or uncertainties. Besides, $\theta_{i i}^{(k)}(t) \in \mathbb{R}^{1}$ and $\theta_{i j}^{(k)}(t) \in \mathbb{R}^{1}$ denote unknown parameters which belong to the following ellipsoidal sets:

$$
\begin{aligned}
& \Delta_{i i} \triangleq\left\{\theta_{i i} \in \mathbb{R}^{p_{i i}} \mid \theta_{i i}^{T}(t) \Sigma_{i i}^{-1} \theta_{i i}(t) \leq 1\right\}, \\
& \Delta_{i j} \triangleq\left\{\theta_{i j} \in \mathbb{R}^{p_{i j}} \quad \mid \quad \theta_{i j}^{T}(t) \Sigma_{i j}^{-1} \theta_{i j}(t) \leq 1\right\} .
\end{aligned}
$$

In (4), $\Sigma_{i i} \in \mathbb{R}^{p_{i i} \times p_{i i}}$ and $\Sigma_{i j} \in \mathbb{R}^{p_{i j} \times p_{i j}}$ represent the size of the ellipsoid and are defined as $\Sigma_{i i}=\operatorname{diag}\left(\sigma_{i i}^{(1)}, \ldots, \sigma_{i i}^{\left(p_{i i}\right)}\right)$ and $\Sigma_{i j}=\operatorname{diag}\left(\sigma_{i j}^{(1)}, \ldots, \sigma_{i j}^{\left(p_{i j}\right)}\right)$, respectively, where $\sigma_{i i}^{(k)}$ and $\sigma_{i j}^{(k)}$ are positive constant; that is, matrices $\Sigma_{i i}$ and $\Sigma_{i j}$ are symmetric 
positive definite. Besides, $\theta_{i i}(t)$ and $\theta_{i j}(t)$ are the unknown parameter vectors given by

$$
\begin{aligned}
\theta_{i i}(t) & =\left(\theta_{i i}^{(1)}(t), \ldots, \theta_{i i}^{\left(p_{i i}\right)}(t)\right)^{T}, \\
\theta_{i j}(t) & =\left(\theta_{i j}^{(1)}(t), \ldots, \theta_{i j}^{\left(p_{i j}\right)}(t)\right)^{T} .
\end{aligned}
$$

Now, the nominal subsystem, ignoring the unknown parameters and the interactions in (2), is given by

$$
\frac{d}{d t} \bar{x}_{i}(t)=A_{i i} \bar{x}_{i}(t)+B_{i} \bar{u}_{i}(t),
$$

where $\bar{x}_{i}(t) \in \mathbb{R}^{n_{i}}$ and $\bar{u}_{i}(t) \in \mathbb{R}^{m_{i}}$ are the vectors of the state and the control input for the $i$ th nominal subsystem, respectively.

First of all, in order to generate the desired trajectory in time response for the uncertain $i$ th subsystem of (2) systematically, we adopt the standard linear quadratic control problem for the $i$ th nominal subsystem of (6). Of course, one can adopt some other design method for deriving the desirable response (e.g., pole placement). It is well known that the optimal control input for the $i$ th nominal subsystem of (6) can be obtained as

$$
\begin{aligned}
& \bar{u}_{i}(t)=K_{i} \bar{x}_{i}(t), \\
& K_{i}=-\mathscr{R}_{i}^{-1} B_{i}^{T} \mathscr{X}_{i} .
\end{aligned}
$$

In (7), $\mathscr{X}_{i} \in \mathbb{R}^{n_{i} \times n_{i}}$ is a symmetric positive define matrix which satisfies the algebraic Riccati equation

$$
H_{e}\left\{A_{i i}^{T} \mathscr{X}_{i}\right\}-\mathscr{X}_{i} B_{i} \mathscr{R}_{i}^{-1} B_{i}^{T} \mathscr{X}_{i}+\mathscr{Q}_{i}=0,
$$

where the weighting matrices $Q_{i} \in \mathbb{R}^{n_{i} \times n_{i}}$ and $\mathscr{R}_{i} \in \mathbb{R}^{m_{i} \times m_{i}}$ are positive definite and are determined in advance so that the desirable transient behavior is achieved.

Based on the existing result [8], we introduce an error vector $e_{i}(t) \triangleq x_{i}(t)-\bar{x}_{i}(t)$. Besides, for the $i$ th subsystem of (2), using the feedback gain matrix $K_{i} \in \mathbb{R}^{m_{i} \times n_{i}}$ of (7), we define the following control input:

$$
u_{i}(t) \triangleq K_{i} x_{i}(t)+v_{i}(t)
$$

where $v_{i}(t) \in \mathbb{R}^{m_{i}}$ is the compensation input $[8,9]$ defined as

$$
v_{i}(t) \triangleq F_{i} e_{i}(t)+\mathscr{L}_{i}\left(x_{i}, e_{i}, t\right) e_{i}(t)
$$

In (10), $F_{i} \in \mathbb{R}^{m_{i} \times n_{i}}$ and $\mathscr{L}_{i}\left(x_{i}, e_{i}, t\right) \in \mathbb{R}^{m_{i} \times n_{i}}$ are the fixed compensation gain matrix and the variable one for the $i$ th subsystem of (2). From (2), (3), (6), (9), and (10), the uncertain error subsystem is derived as

$$
\begin{aligned}
\frac{d}{d t} e_{i}(t)= & \left(A_{K_{i}}+B_{\mathrm{i}} F_{i}\right) e_{i}(t)+\sum_{k=1}^{p_{i i}} \theta_{i}^{(k)}(t) B_{i} \mathscr{E}_{i i}^{(k)} x_{i}(t) \\
& +B_{i} \sum_{\substack{j=1 \\
j \neq i}}^{\mathcal{N}}\left(\mathscr{D}_{i j}+\sum_{k=1}^{p_{i j}} \theta_{i j}^{(k)}(t) \mathscr{E}_{i j}^{(k)}\right) x_{j}(t) \\
& +B_{i} \mathscr{L}_{i}\left(x_{i}, e_{i}, t\right) e_{i}(t) .
\end{aligned}
$$

In (11), $A_{K_{i}} \in \mathbb{R}^{n_{i} \times n_{i}}$ is the stable matrix given by $A_{K_{i}}=A_{i i}+$ $B_{i} K_{i}$.

From the above discussion, our design objective in this paper is to determine the decentralized variable gain robust control of (9) such that the resultant overall system achieves not only robust stability but also satisfactory transient behavior, that is, to design the fixed gain matrix $F_{i} \in \mathbb{R}^{m_{i} \times n_{i}}$ and the variable one $\mathscr{L}_{i}\left(x_{i}, e_{i}, t\right) \in \mathbb{R}^{m_{i} \times n_{i}}$ such that asymptotical stability of the overall error system composed of $\mathcal{N}$ error subsystems of (11) is guaranteed.

\section{Decentralized Variable Gain Controllers}

The following theorem shows sufficient conditions for the existence of the proposed decentralized control system.

Theorem 3. Consider the uncertain error subsystem of (11) and the control input of (9).

By using symmetric positive definite matrices $\mathscr{Y}_{i} \in \mathbb{R}^{n_{i} \times n_{i}}$ and $\mathcal{S}_{i} \in \mathbb{R}^{n_{i} \times n_{i}}$, matrices $\mathscr{W}_{i} \in \mathbb{R}^{m_{i} \times n_{i}}$, and positive constants $\epsilon_{i}$ which satisfy the LMIs

$$
\begin{gathered}
\left(\begin{array}{c|c}
H_{e}\left\{A_{K_{i}} \mathscr{Y}_{i}+B_{i} \mathscr{W}_{i}\right\} & \Lambda\left(\mathscr{Y}_{i}\right) \\
\hline \star & -\Pi\left(\epsilon_{i}\right)
\end{array}\right)<0, \\
\left(\begin{array}{c|c}
H_{e}\left\{\mathcal{S}_{i} A_{K_{i}}\right\} & \Psi_{i} \\
\hline \star & -\Pi\left(\epsilon_{i}\right)
\end{array}\right)<0
\end{gathered}
$$

the fixed gain matrix $F_{i} \in \mathbb{R}^{m_{i} \times n_{i}}$ and the variable one $\mathscr{L}_{i}\left(x_{i}, e_{i}, t\right) \in \mathbb{R}^{m_{i} \times n_{i}}$ are determined as $F_{i}=\mathscr{W}_{i} \mathscr{Y}_{i}^{-1}$ and (14), respectively:

$$
\mathscr{L}_{i}\left(x_{i}, e_{i}, t\right) \triangleq\left\{\begin{array}{c}
-\frac{\zeta_{i}\left(x_{i}, e_{i}, t\right)+\eta_{i}\left(e_{i}, t\right)}{\left\|B_{i}^{T} \mathscr{P}_{i} e_{i}(t)\right\|^{2}} B_{i}^{T} \mathscr{P}_{i} \\
\mathscr{L}_{i}\left(x_{i}, e_{i}, t_{\epsilon}\right) .
\end{array}\right.
$$

In (12)-(14), matrices $\Lambda\left(\mathcal{Y}_{i}\right), \Psi_{i}, \Pi\left(\epsilon_{i}\right), \Gamma\left(x_{i}, t\right), \zeta\left(x_{i}, e_{i}, t\right)$, and $\eta\left(e_{i}, t\right)$ are given by

$$
\begin{aligned}
\Lambda\left(\mathscr{Y}_{i}\right) \triangleq\left(\begin{array}{llllll}
\mathscr{Y}_{i} \mathscr{D}_{1 i}^{T} & \mathscr{Y}_{i} \mathscr{G}_{1 i}^{T} & \cdots & \mathscr{Y}_{i} \mathscr{D}_{i-1 i}^{T} & \mathscr{Y}_{i} \mathscr{G}_{i-1 i}^{T} \\
\mathscr{Y}_{i} \mathscr{D}_{i+1 i}^{T} & \mathscr{Y}_{i} \mathscr{G}_{i+1 i}^{T} & \cdots & \mathscr{Y}_{i} \mathscr{D}_{\mathcal{N} i}^{T} & \mathscr{Y}_{i} \mathscr{G}_{\mathcal{N} i}^{T}
\end{array}\right),
\end{aligned}
$$

$$
\begin{aligned}
& \Psi_{i} \triangleq\left(\begin{array}{lllll}
\mathscr{D}_{1 i}^{T} & \mathscr{G}_{1 i}^{T} & \cdots & \mathscr{D}_{i-1 i}^{T} & \mathscr{G}^{T} T \\
\end{array}\right. \\
& \left.\mathscr{D}_{i+1 i}^{T} \quad \mathscr{G}_{i+1 i}^{T} \cdots \mathscr{D}_{\mathcal{N i}}^{T} \quad \mathscr{G}_{\mathcal{N i}}^{T}\right), \\
& \Pi\left(\epsilon_{i}\right) \triangleq \operatorname{diag}\left(\Pi_{1}\left(\epsilon_{1}\right), \Pi_{2}\left(\epsilon_{2}\right), \ldots,\right. \\
& \Pi_{i-1}\left(\epsilon_{i-1}\right), \Pi_{i+1}\left(\epsilon_{i+1}\right), \ldots, \\
& \left.\Pi_{\mathcal{N}}\left(\epsilon_{\mathcal{N}}\right)\right)
\end{aligned}
$$




$$
\begin{aligned}
& \Pi_{k}\left(\epsilon_{k}\right) \triangleq \operatorname{diag}\left(\epsilon_{k} I_{m_{k}}, \epsilon_{k} I_{q_{k}}\right) \quad(k=1, \ldots, \mathcal{N}), \\
& \Gamma_{i i}\left(x_{i}, t\right) \triangleq\left(\begin{array}{lll}
\mathscr{E}_{i i}^{(1)} x_{i}(t) & \cdots & \mathscr{E}_{i i}^{\left(p_{i i}\right)} x_{i}(t)
\end{array}\right), \\
& \zeta_{i}\left(x_{i}, e_{i}, t\right)=\left\|\sum_{i i}^{1 / 2} \Gamma_{i i}^{T}\left(x_{i}, t\right) B_{i}^{T} \mathscr{P}_{i} e_{i}(t)\right\|, \\
& \eta_{i}\left(e_{i}, t\right)=\epsilon_{i}\left(1+\sigma_{i j}^{*}\right)(\mathcal{N}-1)\left\|B_{i}^{T} \mathscr{P}_{i} e_{i}(t)\right\|^{2},
\end{aligned}
$$

where the matrices $\mathscr{G}_{i j}$ in (15) and scalars $\sigma_{i j}^{*}$ with appropriate dimensions in (18) are given by

$$
\begin{aligned}
& \sigma_{i j}^{*} \triangleq \sum_{\substack{j=1 \\
j \neq i}}^{\mathcal{N}} \sum_{k=1}^{p_{i j}} \sigma_{i j}^{(k)}, \\
& \mathscr{G}_{i j}^{T} \mathscr{G}_{i j} \triangleq \sum_{k=1}^{p_{i j}}\left(\mathscr{E}_{i j}^{(k)}\right)^{T} \mathscr{E}_{i j}^{(k)}
\end{aligned}
$$

Note that since the matrix $\left(\mathscr{E}_{i j}^{(k)}\right)^{T} \mathscr{E}_{i j}^{(k)}$ is symmetric semipositive definite, there always exist the matrices $\mathscr{G}_{i j}$. Besides, $t_{\epsilon}$ in (14) is given by $t_{\epsilon}=\lim _{\epsilon>0, \epsilon \rightarrow 0}(t-\epsilon)$ [7].

Then the overall error system composed of the $\mathcal{N}$ error subsystems of (11) is robustly stable.

Proof. In order to prove Theorem 3, let us define the following quadratic function:

$$
\mathscr{V}(t) \triangleq \sum_{i=1}^{\mathcal{N}} \mathscr{V}_{e_{i}}(t)+\sum_{i=1}^{\mathcal{N}} \mathscr{V}_{\bar{x}_{i}}(t)
$$

where $\mathscr{V}_{e_{i}}(t)$ and $\mathscr{V}_{\bar{x}_{i}}(t)$ are given by

$$
\begin{aligned}
& \mathscr{V}_{e_{i}}(t) \triangleq e_{i}^{T}(t) \mathscr{P}_{i} e_{i}(t), \\
& \mathscr{V}_{\bar{x}_{i}}(t) \triangleq \bar{x}_{i}^{T}(t) \mathcal{S}_{i} \bar{x}_{i}(t) .
\end{aligned}
$$

Since the state vector $x_{i}(t)$ can be written as $x_{i}(t)=e_{i}(t)+$ $\bar{x}_{i}(t)$, we can obtain the following relation for the time derivative of the quadratic functions $\mathscr{V}_{e_{i}}(t)$ of (24):

$$
\begin{aligned}
& \frac{d}{d t} \mathscr{V}_{e_{i}}(t)= e_{i}^{T}(t)\left[H_{e}\left\{\left(A_{K_{i}}+B_{i} F_{i}\right)^{T} \mathscr{P}_{i}\right\}\right] e_{i}(t) \\
&+2 e_{i}^{T}(t) \mathscr{P}_{i}\left(\sum_{k=1}^{p_{i i}} \theta_{i}^{(k)}(t) B_{i} \mathscr{E}_{i i}^{(k)} x_{i}(t)\right) \\
&+2 e_{i}^{T}(t) \mathscr{P}_{i} B_{i}\left\{\sum_{\substack{j=1 \\
j \neq i}}^{\mathcal{N}}\left(\mathscr{D}_{i j}+\sum_{k=1}^{p_{i j}} \theta_{i j}^{(k)}(t) \mathscr{E}_{i j}^{(k)}\right)\right. \\
&\left.\times\left(e_{j}(t)+\bar{x}_{j}(t)\right)\right\}
\end{aligned}
$$

$$
\begin{aligned}
& +2 e_{i}^{T}(t) \mathscr{P}_{i} B_{i} \mathscr{L}_{i}(t) e_{i}(t) \\
= & e_{i}^{T}(t)\left[H_{e}\left\{\left(A_{K_{i}}+B_{i} F_{i}\right)^{T} \mathscr{P}_{i}\right\}\right] e_{i}(t) \\
& +2 e_{i}^{T}(t) \mathscr{P}_{i} B_{i} \Gamma_{i i}\left(x_{i}, t\right) \theta_{i i}(t) \\
& +2 e_{i}^{T}(t) \mathscr{P}_{i} B_{i}\left(\sum_{\substack{j=1 \\
j \neq i}}^{\mathcal{N}} \mathscr{D}_{i j}\left(e_{j}(t)+\bar{x}_{j}(t)\right)\right) \\
& \left.+2 e_{i}^{T}(t) \mathscr{P}_{i} B_{i} \sum_{\mathcal{N}} p_{i j}{ }^{(k)}(t) \mathscr{E}_{i j}^{(k)}\left(e_{j}(t)+\bar{x}_{j}(t)\right)\right\} \\
& \times\left\{\sum_{\substack{j=1 \\
j \neq i}}^{\sum_{k=1} \theta_{i j}}\right\} \\
& +2 e_{i}^{T}(t) \mathscr{P}_{i} B_{i} \mathscr{L}_{i}(t) e_{i}(t) .
\end{aligned}
$$

Besides, by using Lemma 1, assumptions of (4) for the unknown parameters $\theta_{i i}(t)$ and $\theta_{i j}(t)$, and the well-known inequality for any vectors $\alpha$ and $\beta$ with appropriate dimensions and a positive scalar $\delta$

$$
2 \alpha^{T} \beta \leq \delta \alpha^{T} \alpha+\frac{1}{\delta} \beta^{T} \beta,
$$

we have the following relation:

$$
\begin{aligned}
\frac{d}{d t} \mathscr{V}_{e_{i}}(t) \leq & e_{i}^{T}(t)\left[H_{e}\left\{\left(A_{K_{i}}+B_{i} F_{i}\right)^{T} \mathscr{P}_{i}\right\}\right] e_{i}(t) \\
& +2\left\|\sum_{i i}^{1 / 2} \Gamma_{i i}^{T}\left(x_{i}, t\right) B_{i}^{T} \mathscr{P}_{i} e_{i}(t)\right\| \\
& +2 \epsilon_{i}(\mathcal{N}-1) e_{i}^{T}(t) \mathscr{P}_{i} B_{i} B_{i}^{T} \mathscr{P}_{i} e_{i}(t) \\
& +2 \sigma_{i j}^{*} \epsilon_{i}(\mathcal{N}-1) e_{i}^{T}(t) \mathscr{P}_{i} B_{i} B^{T} \mathscr{P}_{i} e_{i}(t) \\
& +\frac{1}{\epsilon_{i}} \sum_{\substack{j=1 \\
j \neq i}}^{\mathcal{N}} e_{j}^{T}(t) \mathscr{D}_{i j}^{T} \mathscr{D}_{i j} e_{j}(t) \\
& +\frac{1}{\epsilon_{i}} \sum_{\substack{j=1 \\
j \neq i}}^{\mathcal{N}} e_{j}^{T}(t)\left\{\sum_{k=1}^{p_{i j}}\left(\mathscr{C}_{i j}^{(k)}\right)^{T} \mathscr{E}_{i j}^{(k)}\right\} e_{j}(t) \\
& +\frac{1}{\epsilon_{i}} \sum_{\substack{j=1 \\
j \neq i}}^{\mathcal{N}} \bar{x}_{j}^{T}(t) \mathscr{D}_{i j}^{T} \mathscr{D}_{i j} \bar{x}_{j}(t) \\
& +\frac{1}{\epsilon_{i}} \sum_{\substack{j=1 \\
j \neq i}}^{\mathcal{N}} \bar{x}_{j}^{T}(t)\left\{\sum_{k=1}^{p_{i j}}\left(\mathscr{E}_{i j}^{(k)}\right)^{T} \mathscr{C}_{i j}^{(k)}\right\} \bar{x}_{j}(t) \\
& +2 e_{i}^{T}(t) \mathscr{P}_{i} B_{i} \mathscr{L}_{i}(t) e_{i}(t) .
\end{aligned}
$$


Therefore from the definition of the matrices $\mathscr{G}_{i j}$ in (22), the inequality of (28) can be rewritten as

$$
\begin{aligned}
\frac{d}{d t} \mathscr{V}_{e_{i}}(t) \leq & e_{i}^{T}(t)\left[H_{e}\left\{\left(A_{K_{i}}+B_{i} \mathrm{~F}_{i}\right)^{T} \mathscr{P}_{i}\right\}\right] e_{i}(t) \\
& +2\left\|\sum_{i i}^{1 / 2} \Gamma_{i i}^{T}\left(x_{i}, t\right) B_{i}^{T} \mathscr{P}_{i} e_{i}(t)\right\| \\
& +2 \epsilon_{i}(\mathcal{N}-1) e_{i}^{T}(t) \mathscr{P}_{i} B_{i} B_{i}^{T} \mathscr{P}_{i} e_{i}(t) \\
& +2 \sigma_{i j}^{*} \epsilon_{i}(\mathscr{N}-1) e_{i}^{T}(t) \mathscr{P}_{i} B_{i} B^{T} \mathscr{P}_{i} e_{i}(t) \\
& +\frac{1}{\epsilon_{i}} \sum_{\substack{j=1 \\
j \neq i}}^{\mathcal{N}} e_{j}^{T}(t)\left(\mathscr{D}_{i j}^{T} \mathscr{D}_{i j}+\mathscr{G}_{i j}^{T} \mathscr{G}_{i j}\right) e_{j}(t) \\
& +\frac{1}{\epsilon_{i}} \sum_{\substack{j=1 \\
j \neq i}}^{\mathcal{N}} \bar{x}_{j}^{T}(t)\left(\mathscr{D}_{i j}^{T} \mathscr{D}_{i j}+\mathscr{G}_{i j}^{T} \mathscr{G}_{i j}\right) \bar{x}_{j}(t) \\
& +2 e_{i}^{T}(t) \mathscr{P}_{i} B_{i} \mathscr{L}_{i}(t) e_{i}(t) .
\end{aligned}
$$

Furthermore, one can easily see that the following relation for the quadratic functions $\mathscr{V}_{\bar{x}_{i}}(t)$ of $(25)$ is satisfied:

$$
\frac{d}{d t} \mathscr{V}_{\bar{x}_{i}}(t)=\bar{x}_{i}^{T}(t)\left[H_{e}\left\{A_{K_{i}}^{T} \mathcal{S}_{i}\right\}\right] \bar{x}_{i}(t)
$$

Firstly, we consider the case of $B_{i}^{T} \mathscr{P}_{i} e_{i}(t) \neq 0$. In this case, substituting the variable gain matrix of (14) into (29) and some algebraic manipulations give the inequality

$$
\begin{aligned}
\frac{d}{d t} \mathscr{V}_{e_{i}}(t) \leq & e_{i}^{T}(t)\left[H_{e}\left\{\left(A_{K_{i}}+B_{i} F_{i}\right)^{T} \mathscr{P}_{i}\right\}\right] e_{i}(t) \\
& +\frac{1}{\epsilon_{i}} \sum_{\substack{j=1 \\
j \neq i}}^{\mathcal{N}} e_{j}^{T}(t)\left(\mathscr{D}_{i j}^{T} \mathscr{D}_{i j}+\mathscr{G}_{i j}^{T} \mathscr{G}_{i j}\right) e_{j}(t) \\
& +\frac{1}{\epsilon_{i}} \sum_{\substack{j=1 \\
j \neq i}}^{\mathcal{N}} \bar{x}_{j}^{T}(t)\left(\mathscr{D}_{i j}^{T} \mathscr{D}_{i j}+\mathscr{G}_{i j}^{T} \mathscr{G}_{i j}\right) \bar{x}_{j}(t) .
\end{aligned}
$$

Thus, from the definition of the quadratic function $\mathscr{V}(t)$ of (23), the following relation can be obtained:

$$
\begin{aligned}
\frac{d}{d t} \mathscr{V}(t) \leq & \sum_{i=1}^{\mathcal{N}} e_{i}^{T}(t)\left[H_{e}\left\{\left(A_{K_{i}}+B_{i} F_{i}\right)^{T} \mathscr{P}_{i}\right\}\right] e_{i}(t) \\
& +\sum_{i=1}^{\mathcal{N}} \bar{x}_{i}^{T}(t)\left[H_{e}\left\{A_{K_{i}}^{T} \mathcal{S}_{i}\right\}\right] \bar{x}_{i}(t)
\end{aligned}
$$

$$
\begin{aligned}
+\sum_{i=1}^{\mathcal{N}}\left\{\frac{1}{\epsilon_{i}} \sum_{\substack{j=1 \\
j \neq i}}^{\mathcal{N}} e_{j}^{T}(t)\left(\mathscr{D}_{i j}^{T} \mathscr{D}_{i j}+\mathscr{G}_{i j}^{T} \mathscr{G}_{i j}\right) e_{j}(t)\right. \\
\left.+\frac{1}{\epsilon_{i}} \sum_{\substack{j=1 \\
j \neq i}}^{\mathcal{N}} \bar{x}_{j}^{T}(t)\left(\mathscr{D}_{i j}^{T} \mathscr{D}_{i j}+\mathscr{G}_{i j}^{T} \mathscr{G}_{i j}\right) \bar{x}_{j}(t)\right\} .
\end{aligned}
$$

The inequality of (32) can be rewritten as

$$
\begin{aligned}
& \frac{d}{d t} \mathscr{V}(t) \leq \sum_{i=1}^{\mathcal{N}} e_{i}^{T}(t)\left[H_{e}\left\{\left(A_{K_{i}}+B_{i} F_{i}\right)^{T} \mathscr{P}_{i}\right\}\right. \\
& \left.+\sum_{\substack{j=1 \\
j \neq i}}^{\mathcal{N}} \frac{1}{\epsilon_{j}}\left(\mathscr{D}_{j i}^{T} \mathscr{D}_{j i}+\mathscr{G}_{j i}^{T} \mathscr{G}_{j i}\right)\right] e_{i}(t) \\
& +\sum_{i=1}^{\mathcal{N}} \bar{x}_{i}^{T}(t)\left[H_{e}\left\{A_{K_{i}}^{T} \mathcal{S}_{i}\right\}\right. \\
& \left.+\sum_{\substack{j=1 \\
j \neq i}}^{\mathcal{N}} \frac{1}{\epsilon_{j}}\left(\mathscr{D}_{j i}^{T} \mathscr{D}_{j i}+\mathscr{G}_{j i}^{T} \mathscr{G}_{j i}\right)\right] \bar{x}_{i}(t) .
\end{aligned}
$$

If the matrix inequalities

$$
\begin{gathered}
H_{e}\left\{\left(A_{K_{i}}+B_{i} F_{i}\right)^{T} \mathscr{P}_{i}\right\}+\sum_{\substack{j=1 \\
j \neq i}}^{\mathcal{N}} \frac{1}{\epsilon_{j}}\left(\mathscr{D}_{j i}^{T} \mathscr{D}_{j i}+\mathscr{G}_{j i}^{T} \mathscr{G}_{j i}\right)<0 \\
H_{e}\left\{A_{K_{i}}^{T} \mathcal{S}_{i}\right\}+\sum_{\substack{j=1 \\
j \neq i}}^{\mathcal{N}} \frac{1}{\epsilon_{j}}\left(\mathscr{D}_{j i}^{T} \mathscr{D}_{j i}+\mathscr{G}_{j i}^{T} \mathscr{G}_{j i}\right)<0
\end{gathered}
$$

hold, then the following inequality for the time derivative of the quadratic function $\mathscr{V}(t)$ of $(23)$ is satisfied:

$$
\frac{d}{d t} \mathscr{V}(t)<0, \quad \forall \chi(t) \neq 0,
$$

where $\chi(t) \triangleq\left(e_{1}^{T}(t), \ldots, e_{\mathcal{N}}^{T}(t), \bar{x}_{1}^{T}(t), \ldots, \bar{x}_{\mathcal{N}}^{T}(t)\right)^{T}$.

Next we consider the case of $B_{i}^{T} \mathscr{P}_{i} e_{i}(t)=0$. In this case, one can see from (26) and (30) that the time derivative of the quadratic function $\mathscr{V}(t)$ of $(23)$ can be written as

$$
\begin{aligned}
\frac{d}{d t} \mathscr{V}(t)= & \sum_{i=1}^{\mathcal{N}} e_{i}^{T}(t)\left[H_{e}\left\{\left(A_{K_{i}}+B_{i} F_{i}\right)^{T} \mathscr{P}_{i}\right\}\right] e_{i}(t) \\
& +\sum_{i=1}^{\mathcal{N}} \bar{x}_{i}^{T}(t)\left[H_{e}\left\{A_{K_{i}}^{T} \mathcal{S}_{i}\right\}\right] \bar{x}_{i}(t) .
\end{aligned}
$$


Namely, in the case of $B_{i}^{T} \mathscr{P}_{i} e_{i}(t)=0$, the relation of (36) is also satisfied, provided that the inequalities of (34) and (35) hold.

From the above, the overall error system is clearly guaranteed to be stable, because all nominal subsystems are asymptotically stable.

Finally, we consider the inequalities of (34) and (35). By introducing the matrices $\mathscr{Y}_{i} \triangleq \mathscr{P}_{i}^{-1}$ and $\mathscr{W}_{i} \triangleq F_{i} \mathscr{P}_{i}$ and pre- and postmultiplying both sides of the matrix inequality of (34) by $\mathscr{Y}_{i}$, we have the inequality

$$
H_{e}\left\{A_{K_{i}} \mathscr{Y}_{i}+B_{i} \mathscr{W}_{i}\right\}+\sum_{\substack{j=1 \\ j \neq i}}^{\mathcal{N}} \frac{1}{\epsilon_{j}} \mathscr{Y}_{i}\left(\mathscr{D}_{j i}^{T} \mathscr{D}_{j i}+\mathscr{G}_{j i}^{T} \mathscr{G}_{j i}\right) \mathscr{Y}_{i}<0 .
$$

Thus by applying Lemma 2 (Schur complement) to (35) and (38), we find that the inequalities of (35) and (38) are equivalent to the LMIs of (13) and (12), respectively.

Therefore the proof of Theorem 3 is accomplished.

Remark 4. The positive constants $\epsilon_{i}$ in LMIs of (12) and (13) are included in $(2,2)$-block only. Namely, the positive constants $\epsilon_{i}$ are independent of LMI variables $\mathscr{Y}_{i}$ and $\mathscr{W}_{i}$. Thus the positive constants $\epsilon_{i}$ can arbitrarily be selected and one can see that the LMIs of (12) and (13) are always feasible. Thus by using the solution of the LMIs of (12) and (13) the fixed compensation gain matrix is determined as $F_{i}=\mathscr{W}_{i} \mathscr{Y}_{i}^{-1}$ and the variable one is given by (14).

Remark 5. The proposed decentralized robust controller design method is adaptable when some assumptions are satisfied. Namely, if the matching condition for uncertainties and interactions is satisfied, then the LMIs of (12) and (13) are feasible; that is, the proposed decentralized variable gain robust controller is applicable. Besides, in the proposed controller design method, the number of LMIs needed to be solved is $\mathscr{N}$.

On the other hand, the number of the inequalities needed to be solved in the conventional decentralized robust control with fixed gain matrices is " 2 ", provided that the number of unknown parameters included in the overall system is $\mathscr{M}$ (see the appendix). Therefore, one can see that the proposed controller design method is very useful.

\section{Numerical Examples}

In order to demonstrate the efficiency of the proposed robust controller, we have run a simple example. The control problem considered here is not necessarily practical. However, the simulation results stated below illustrate the distinct feature of the proposed decentralized robust controller.

In this example, we consider the uncertain large-scale interconnected systems consisting of three two-dimensional subsystems; that is, $\mathcal{N}=3$. The system parameters are given as follows:

$$
\begin{aligned}
& A_{11}=\left(\begin{array}{rr}
-1.0 & 1.0 \\
0.0 & 1.0
\end{array}\right), \quad A_{22}=\left(\begin{array}{cc}
0.0 & 1.0 \\
-1.0 & -1.0
\end{array}\right), \\
& A_{33}=\left(\begin{array}{cc}
1.0 & 0.0 \\
-1.0 & -3.0
\end{array}\right), \quad B_{1}=\left(\begin{array}{l}
0.0 \\
1.0
\end{array}\right) \text {, } \\
& B_{2}=\left(\begin{array}{l}
1.0 \\
1.0
\end{array}\right), \quad B_{3}=\left(\begin{array}{l}
1.0 \\
0.0
\end{array}\right) \text {, } \\
& \mathscr{E}_{11}^{(1)^{T}}=\left(\begin{array}{l}
1.0 \\
0.0
\end{array}\right), \quad \mathscr{E}_{11}^{(2)^{T}}=\left(\begin{array}{l}
0.0 \\
1.0
\end{array}\right), \\
& \mathscr{E}_{22}^{(1)^{T}}=\left(\begin{array}{l}
1.0 \\
0.0
\end{array}\right), \quad \mathscr{E}_{22}^{(2)^{T}}=\left(\begin{array}{l}
0.0 \\
1.0
\end{array}\right) \text {, } \\
& \mathscr{E}_{33}^{(1)}=\left(\begin{array}{l}
0.0 \\
1.0
\end{array}\right), \quad \mathscr{E}_{33}^{(2)^{T}}=\left(\begin{array}{l}
1.0 \\
0.0
\end{array}\right), \\
& \mathscr{D}_{12}^{T}=\left(\begin{array}{l}
1.0 \\
0.0
\end{array}\right), \quad \mathscr{D}_{13}^{T}=\left(\begin{array}{l}
2.0 \\
1.0
\end{array}\right), \quad \mathscr{D}_{21}^{T}=\left(\begin{array}{l}
1.0 \\
2.0
\end{array}\right), \\
& \mathscr{D}_{23}^{T}=\left(\begin{array}{l}
0.0 \\
3.0
\end{array}\right), \quad \mathscr{D}_{31}^{T}=\left(\begin{array}{l}
2.0 \\
2.0
\end{array}\right), \quad \mathscr{D}_{32}^{T}=\left(\begin{array}{l}
1.0 \\
1.0
\end{array}\right), \\
& \mathscr{E}_{12}^{(1)^{T}}=\left(\begin{array}{l}
1.0 \\
0.0
\end{array}\right), \quad \mathscr{E}_{12}^{(2)^{T}}=\left(\begin{array}{l}
0.0 \\
1.0
\end{array}\right), \quad \mathscr{E}_{13}^{(1)^{T}}=\left(\begin{array}{l}
1.0 \\
0.0
\end{array}\right) \text {, } \\
& \mathscr{E}_{13}^{(2)}=\left(\begin{array}{l}
0.0 \\
1.0
\end{array}\right), \quad \mathscr{E}_{21}^{(1)^{T}}=\left(\begin{array}{l}
1.0 \\
0.0
\end{array}\right), \quad \mathscr{E}_{21}^{(2)^{T}}=\left(\begin{array}{l}
0.0 \\
1.0
\end{array}\right), \\
& \mathscr{E}_{23}^{(1)}=\left(\begin{array}{l}
1.0 \\
0.0
\end{array}\right), \quad \mathscr{E}_{23}^{(2)^{T}}=\left(\begin{array}{l}
0.0 \\
1.0
\end{array}\right), \quad \mathscr{E}_{31}^{(1)^{T}}=\left(\begin{array}{l}
0.0 \\
1.0
\end{array}\right) \\
& \mathscr{E}_{31}^{(2)}=\left(\begin{array}{l}
1.0 \\
0.0
\end{array}\right), \quad \mathscr{E}_{32}^{(1)^{T}}=\left(\begin{array}{l}
0.0 \\
1.0
\end{array}\right), \quad \mathscr{E}_{32}^{(2)^{T}}=\left(\begin{array}{l}
0.0 \\
1.0
\end{array}\right) .
\end{aligned}
$$

Firstly, we choose the weighting matrices $Q_{i} \in \mathbb{R}^{2 \times 2}$ and $\mathscr{R}_{i} \in \mathbb{R}^{1 \times 1}(i=1, \ldots, 3)$ for the nominal subsystems such as $Q_{1}=\operatorname{diag}(1.0,2.0), Q_{2}=\operatorname{diag}\left(1.0,1.0 \times 10^{1}\right), Q_{3}=I_{3}, \mathscr{R}_{1}=$ 1.0, $\mathscr{R}_{2}=1.0 \times 10^{1}$, and $\mathscr{R}_{3}=1.0 \times 10^{1}$. Besides, the design parameters $\Sigma_{i i}$ and $\Sigma_{i j}$ are selected as $\Sigma_{i i}=\operatorname{diag}(3.0,2.0)$ and $\Sigma_{i j}=\operatorname{diag}(4.0,3.0)$. By solving the algebraic Riccati equation of (8) the following optimal gain matrices $K_{i} \in \mathbb{R}^{1 \times 2}$ for the $i$ th nominal subsystem are derived:

$$
\begin{aligned}
& K_{1}=\left(\begin{array}{ll}
-1.71572 \times 10^{-1}-2.82843
\end{array}\right), \\
& K_{2}=(-4.45866-3.42538) \times 10^{-1}, \\
& K_{3}=\left(\begin{array}{ll}
-2.052724 .11182 \times 10^{-3}
\end{array}\right) .
\end{aligned}
$$

Next, by using Theorem 3 we design the proposed decentralized variable gain robust controller. By solving LMIs of 
(12) and (13), we have positive definite matrices $\mathscr{Y}_{i} \in \mathbb{R}^{2 \times 2}$, matrices $\mathscr{W}_{i} \in \mathbb{R}^{1 \times 2}$, and positive scalars $\epsilon_{i}$ given by

$$
\begin{aligned}
& \mathscr{Y}_{1}=\left(\begin{array}{cc}
6.1524 & -2.8845 \\
\star & 1.1481 \times 10^{1}
\end{array}\right), \\
& \mathscr{Y}_{2}=\left(\begin{array}{cc}
3.7768 & 1.5321 \\
\star & 5.3977
\end{array}\right), \\
& \mathscr{Y}_{3}=\left(\begin{array}{cc}
7.4690 & -1.8692 \\
\star & 7.1578
\end{array}\right), \\
& \mathscr{W}_{1}^{T}=\left(\begin{array}{c}
-1.2512 \times 10^{1} \\
-1.8741
\end{array}\right), \\
& \mathscr{W}_{2}^{T}=\left(\begin{array}{c}
-8.6852 \\
-8.9201
\end{array}\right) \\
& \mathscr{W}_{3}^{T}=\left(\begin{array}{c}
-1.5089 \times 10^{1} \\
-2.1731 \times 10^{1}
\end{array}\right), \\
& \epsilon_{1}=1.5697 \times 10^{1}, \\
& \epsilon_{2}=1.2076 \times 10^{1}, \\
& \epsilon_{3}=2.8810 \times 10^{1} .
\end{aligned}
$$

Thus the fixed gain matrices $F_{i} \in \mathbb{R}^{1 \times 2}$ can be computed as

$$
\begin{aligned}
& F_{1}=\left(-2.3919-7.6416 \times 10^{-1}\right), \\
& F_{2}=(-1.8413-1.1300), \\
& F_{3}=(-2.9744-3.8127) .
\end{aligned}
$$

Note that the number of LMIs needed to be solved in the conventional decentralized robust control with a fixed gain matrix is $2^{9}=512$ (see the appendix). Namely, it is difficult to design the conventional fixed gain decentralized robust controller comparing with the proposed design method of the decentralized variable gain robust control.

The result of this example is shown in Figures 1, 2, 3, and 4. In this example, initial values of the uncertain large-scale system with system parameters of (39)-(42) and the nominal system are selected as follows:

$$
\begin{aligned}
& x(0)=\left(\begin{array}{ll|ll|ll}
1.0 & -1.0 & 2.0 & 0.0 & 1.0 & -2.0
\end{array}\right)^{T}, \\
& \bar{x}(0)=\left(\begin{array}{ll|ll|ll}
1.0 & -1.5 & 2.25 & 0.0 & 1.5 & -1.5
\end{array}\right)^{T} .
\end{aligned}
$$

In these figures, $x_{i}^{(l)}(t)$ and $\bar{x}_{i}^{(l)}(t)$ denote the $l$ th element of $x_{i}(t)$ for $i$ th subsystem and one of the state $\bar{x}_{i}(t)$ for $i$ th nominal subsystem, respectively. Furthermore, unknown parameters are given as

$$
\begin{aligned}
& \theta_{i i}(t)=\left(\begin{array}{ll}
\sqrt{3.0} \sin (5.0 \pi t) & -\sqrt{2.0} \cos (2.0 \pi t)
\end{array}\right)^{T}, \\
& \theta_{i j}(t)=\left(\begin{array}{ll}
2.0 \cos (3.0 \pi t) & \sqrt{3.0} \sin (4.0 \pi t)
\end{array}\right)^{T} .
\end{aligned}
$$

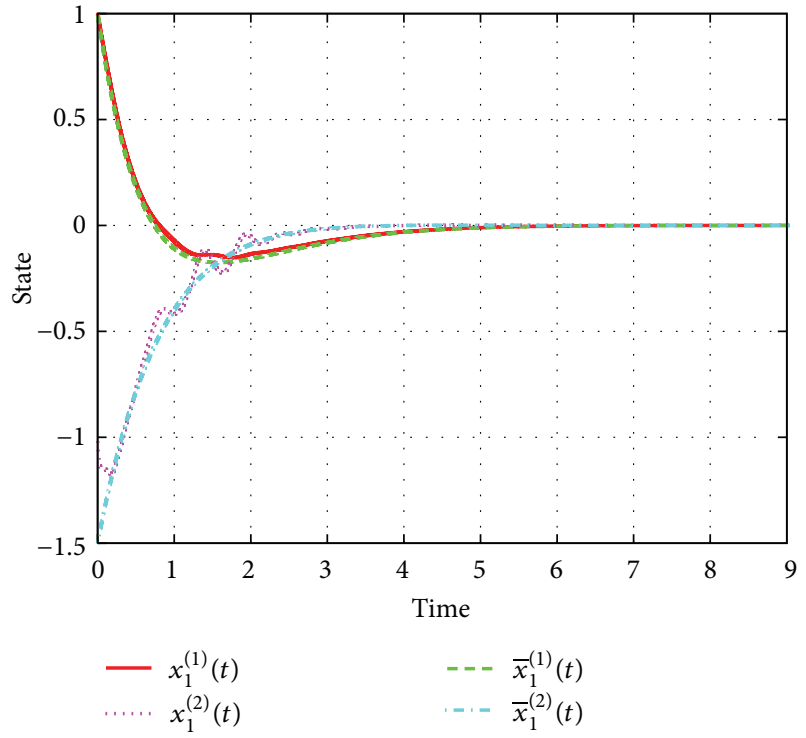

FIgURE 1: Time histories of $x_{1}(t)$ and $\bar{x}_{1}(t)$.

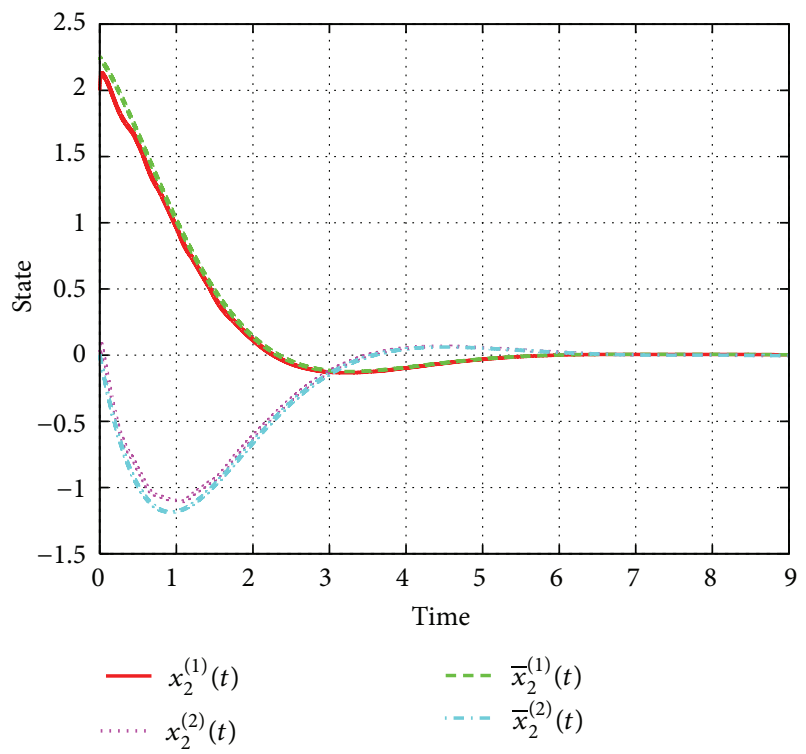

Figure 2: Time histories of $x_{2}(t)$ and $\bar{x}_{2}(t)$.

From these figures, the proposed decentralized variable gain controller stabilizes the uncertain large-scale systems with system parameters of (39)-(42) in spite of uncertainties and interactions. Besides, the proposed variable gain robust control system achieves good transient response close to desired transient behavior generated by the nominal subsystem. Therefore, the effectiveness of the proposed decentralized robust control system has been shown.

\section{Conclusions}

In this paper, on the basis of the work of Oya and Hagino [8], we have suggested a decentralized variable gain robust 


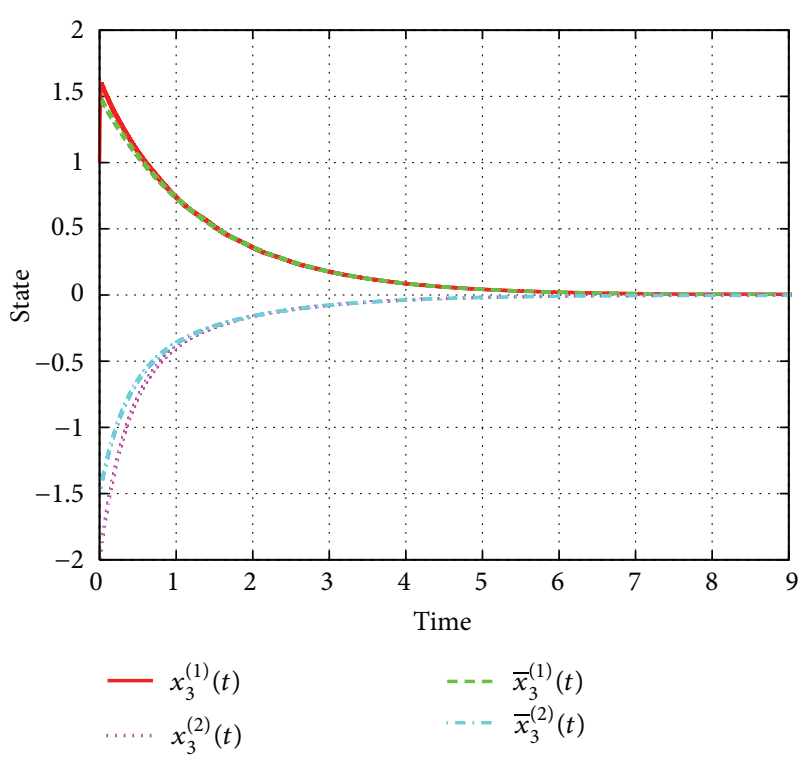

Figure 3: Time histories of $x_{3}(t)$ and $\bar{x}_{3}(t)$.

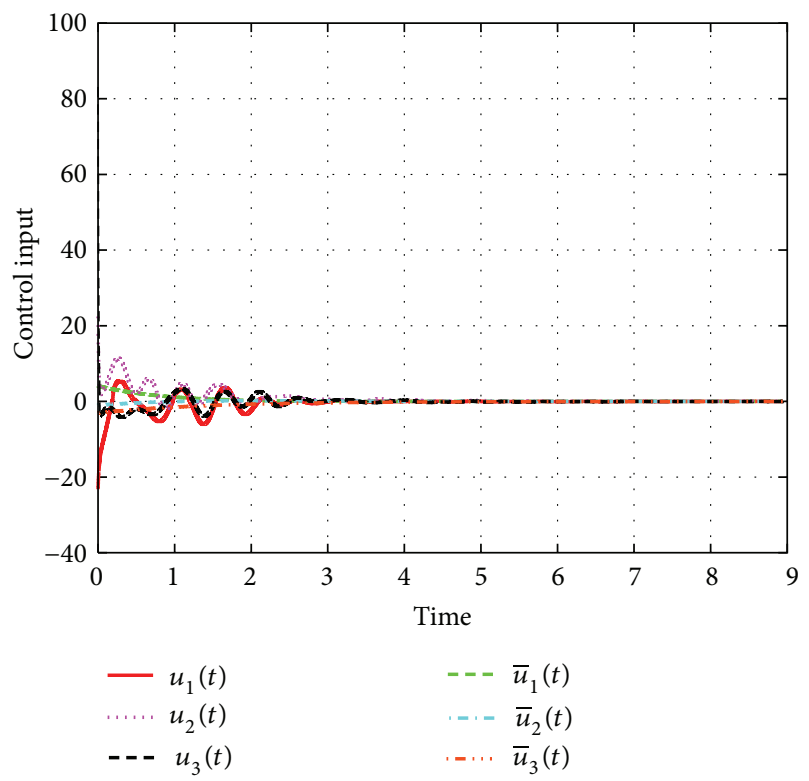

FIgURE 4: Time histories of $u_{i}(t)$ and $\bar{u}_{i}(t)$.

controller for a large-scale interconnected system with uncertainties. For the uncertain large-scale interconnected systems, we have presented an LMI-based design method of the proposed decentralized variable gain robust controller. In addition, the effectiveness of the proposed decentralized robust controller has been shown by simple numerical examples. One can easily find that since the parameters $\epsilon_{i}$ in LMIs of (12) and (13) can arbitrarily be selected, these LMIs can be easily solved. Namely, the proposed design approach is very useful comparing with the conventional decentralized robust control.
In the future research, we will extend the proposed controller synthesis to such a broad class of systems as largescale systems with general uncertainties, uncertain largescale systems with time delays, and so on.

\section{Appendix}

In this appendix, we show an LMI-based design method of the conventional decentralized robust controller with a fixed gain matrix for the uncertain large-scale interconnected systems of (2).

We consider the overall system composed of the $\mathcal{N}$ subsystems of (2). Since the $i$ th subsystem is given by (2), we find that the overall system can be written as

$$
\frac{d}{d t} x(t)=\mathscr{A}(\theta) x(t)+\mathscr{B} u(t)
$$

where $x(t)=\left(x_{1}^{T}(t), \ldots, x_{\mathcal{N}}^{T}(t)\right)^{T}$. In (A.2), the system matrix $\mathscr{A}(t) \in \mathbb{R}^{n \times n}$ and the input one $\mathscr{B} \in \mathbb{R}^{n \times m}$ ( $n$ and $m$ are given by $n=\sum_{i=1}^{\mathcal{N}} n_{i}$ and $m=\sum_{i=1}^{\mathcal{N}} m_{i}$, resp.) are given by

$$
\begin{gathered}
\mathscr{A}(\theta)=\left(\begin{array}{cccc}
A_{11}\left(\theta_{11}\right) & A_{12}\left(\theta_{12}\right) & \cdots & A_{1 \mathcal{N}}\left(\theta_{1 \mathscr{N}}\right) \\
A_{21}\left(\theta_{21}\right) & A_{22}\left(\theta_{22}\right) & \cdots & A_{2 \mathcal{N}}\left(\theta_{2 \mathscr{N}}\right) \\
\vdots & \vdots & \ddots & \vdots \\
A_{\mathcal{N} 1}\left(\theta_{\mathcal{N} 1}\right) & A_{\mathcal{N} 2}\left(\theta_{\mathcal{N} 2}\right) & \cdots & A_{\mathcal{N N}}\left(\theta_{\mathcal{N N}}\right)
\end{array}\right), \\
\mathscr{B}=\operatorname{diag}\left(B_{1}, B_{2}, \cdots, B_{\mathcal{N}}\right) .
\end{gathered}
$$

One can easily see that the system matrix $\mathscr{A}(\theta)$ affinely depends on the unknown parameters $\theta_{i i}(t)$ and $\theta_{i j}(t)$, because the matrices $A_{i i}(t)$ and $A_{i j}(t)$ are given by (3). Besides, the number of unknown parameters in the overall system of (A.1) can be computed as

$$
\mathscr{M} \triangleq \sum_{i=1}^{\mathcal{N}}\left(p_{i i}+\sum_{j=1, j \neq i}^{\mathcal{N}} p_{i j}\right) .
$$

For the overall system of (A.1), we consider the following decentralized robust control input for the $i$ th subsystem:

$$
u_{i}(t)=K_{\mathscr{C}_{i}} x_{i}(t),
$$

where $K_{\mathscr{C}_{i}} \in \mathbb{R}^{m_{i} \times n_{i}}$ is a fixed gain matrix for the $i$ th subsystem. Then the closed-loop system can be rewritten as

$$
\frac{d}{d t} x(t)=(\mathscr{A}(t)+\mathscr{B} \mathscr{K}) x(t)
$$

where the fixed gain $\mathscr{K} \in \mathbb{R}^{m \times n}$ is given by

$$
\mathscr{K}=\operatorname{diag}\left(K_{\mathscr{C}_{1}}, K_{\mathscr{C}_{2}}, \ldots, K_{\mathscr{C}_{\mathscr{N}}}\right) \text {. }
$$

Therefore the problem is to derive the fixed gain matrices $K_{\mathscr{C}_{i}}$ such that the closed-loop system of (A.5) is robustly stable.

In order to design the fixed gain matrices $K_{\mathscr{C}_{i}} \in \mathbb{R}^{m_{i} \times n_{i}}$, we consider the quadratic function

$$
\mathscr{V}_{\mathscr{K}}(t) \triangleq x^{T}(t) \mathscr{X}_{\mathscr{K}} x(t) .
$$


In (A.7), $\mathscr{X}_{\mathscr{K}} \in \mathbb{R}^{n \times n}$ is a symmetric positive definite matrix defined as $\mathscr{X}_{\mathscr{K}}=\operatorname{diag}\left(\mathscr{X}_{\mathscr{K}_{1}}, \ldots, \mathscr{X}_{\mathscr{K}_{\mathscr{N}}}\right)$ where $\mathscr{X}_{\mathscr{K}_{i}} \in \mathbb{R}^{n_{i} \times n_{i}}$.

The time derivative of the quadratic function $\mathscr{V}_{\mathscr{K}}(t)$ of (A.7) along the trajectory of the closed-loop system of (A.5) can be written as

$$
\frac{d}{d t} \mathscr{V}_{\mathscr{K}}(t)=x^{T}(t)\left[H_{e}\left\{\mathscr{X}_{\mathscr{K}}(\mathscr{A}(\theta)+\mathscr{B} \mathscr{K})\right\}\right] x(t) .
$$

Namely, if the following relation is satisfied, then the robust stability of the closed-loop system of (A.5) is guaranteed:

$$
H_{e}\left\{\mathscr{X}_{\mathscr{K}}(\mathscr{A}(\theta)+\mathscr{B} \mathscr{K})\right\}<0, \quad \forall \theta_{i i}(t) \in \Delta_{i i}, \forall \theta_{i j}(t) \in \Delta_{i j}
$$

By introducing two complementary variables $\mathscr{Z}_{i}=\mathscr{X}_{\mathscr{K}_{i}}^{-1}$ and $\mathscr{U}_{i}=K_{\mathscr{C}_{i}} \mathscr{Z}_{i}$ and pre- and postmultiplying (A.9) by $\mathscr{Z}=\operatorname{diag}\left(\mathscr{Z}_{1}, \ldots, \mathscr{Z}_{\mathscr{N}}\right)$, the condition (A.9) is written as

$$
H_{e}\{\mathscr{A}(\theta) \mathscr{Z}+\mathscr{B} \mathscr{U}\}<0, \quad \forall \theta_{i i}(t) \in \Delta_{i i}, \forall \theta_{i j}(t) \in \Delta_{i j},
$$

where $\mathscr{U}=\operatorname{diag}\left(\mathscr{U}_{1}, \ldots, \mathscr{U}_{\mathcal{N}}\right)$.

Now, we define the following unknown parameter vector:

$$
\begin{aligned}
\delta(t) \triangleq & \left(\delta_{1}(t), \ldots, \delta_{\mathscr{M}}(t)\right) \\
= & \left(\theta_{11}^{T}(t), \theta_{12}^{T}(t), \ldots, \theta_{1 \mathcal{N}}^{T}(t) \ldots,\right. \\
& \theta_{21}^{T}(t), \theta_{22}^{T}(t), \ldots, \theta_{\mathcal{N} 2}^{T}(t), \ldots, \\
& \left.\theta_{\mathcal{N} 1}^{T}(t), \theta_{\mathcal{N} 2}^{T}(t), \ldots, \theta_{\mathcal{N N}}^{T}(t)\right) .
\end{aligned}
$$

Next, by introducing the constant vector

$$
\begin{aligned}
\xi \triangleq & \left(\xi_{1}, \ldots, \xi_{\mathscr{M}}\right) \\
= & \left(\sigma_{11}^{(1)}, \ldots, \sigma_{11}^{\left(p_{11}\right)}, \sigma_{12}^{(1)}, \ldots, \sigma_{12}^{\left(p_{12}\right)}, \ldots,\right. \\
& \left.\sigma_{\mathscr{N} 1}^{(1)}, \ldots, \sigma_{\mathcal{N} 1}^{\left(p_{\mathcal{N} 1}\right)}, \ldots, \sigma_{\mathcal{N} \mathcal{N}}^{(1)}, \ldots, \sigma_{\mathcal{N} \mathcal{N}}^{\left(p_{\mathcal{N}}\right)}\right)
\end{aligned}
$$

we consider the bounded set expressed by the hypercube

$$
\bar{\Delta} \triangleq\left\{\delta \in \mathbb{R}^{\mathscr{M}}|| \delta_{l}(t) \mid \leq \xi_{l}\right\} \quad(l=1, \ldots, \mathscr{M}) .
$$

One can easily see that the unknown parameter vector $\delta(t)$ belongs to the hypercube of (A.13). Thus, by considering the sets of $2^{\mathscr{M}}$ vertices of the hypercube $\bar{\Delta}$ such as

$$
\Delta_{\mathrm{vex}} \triangleq\left\{\delta \in \mathfrak{R}^{\mathscr{M}} \mid \delta_{l}=\xi_{l} \text { or } \delta_{l}=-\xi_{l}\right\}
$$

the design problem of the fixed gain matrices $K_{\mathscr{C}_{i}}$ is one of finding the matrices $\mathscr{X}_{\mathscr{K}_{i}}$ and $Y_{\eta}$ which satisfy the condition

$$
H_{e}\{\mathscr{A}(\delta) \mathscr{Z}+\mathscr{B} \mathscr{U}\}<0, \quad \forall \delta \in \Delta_{\text {vex }} .
$$

From the above if the LMIs of (A.15) are feasible, then by using the solution $\mathscr{Z} \in \mathbb{R}^{n \times n}$ and $\mathscr{Z} \in \mathbb{R}^{m \times n}$ the fixed gain matrices $K_{\mathscr{C}_{i}}$ can be obtained as

$$
K_{\mathscr{C}_{i}}=\mathscr{U}_{i} \mathscr{Z}_{i}^{-1}
$$

Consequently, the following theorem for designing the conventional decentralized robust control system is obtained. Theorem A.1. Consider the large-scale interconnected system of (2) and the decentralized control input of (A.4).

If there exist the symmetric positive definite matrix $\mathscr{Z}=$ $\operatorname{diag}\left(\mathscr{X}_{1}, \ldots, \mathscr{X}_{\mathcal{N}}\right)$ and the matrix $\mathcal{U}=\operatorname{diag}\left(\mathcal{U}_{1}, \ldots, \mathscr{U}_{\mathcal{N}}\right)$ which satisfy the LMIs of (A.15), then the fixed gain matrix $K_{\mathscr{C}_{i}}$ for the decentralized control input of (A.4)can be computed as (A.16).

\section{Conflict of Interests}

The authors declare that there is no conflict of interests regarding the publication of this paper.

\section{References}

[1] K. Zhou, J. C. Doyle, and K. Glover, Robust and Optimal Control, Prentice Hall, 1996.

[2] K. Zhou, Essentials of Robust Control, Prentice Hall, 1998.

[3] I. R. Petersen, "A Riccati equation approach to the design of stabilizing controllers and observers for a class of uncertain linear systems," IEEE Transactions on Automatic Control, vol. 30, no. 9, pp. 904-907, 1985.

[4] W. E. Schmitendorf, "Designing stabilizing controllers for uncertain systems using the Riccati equation approach," IEEE Transactions on Automatic Control, vol. 33, no. 4, pp. 376-379, 1988.

[5] J. C. Doyle, K. Glover, P. P. Khargonekar, and B. A. Francis, "State-space solutions to standard $H_{2}$ and $H_{\infty}$ control problems," IEEE Transactions on Automatic Control, vol. 34, no. 8, pp. 831-847, 1989.

[6] P. P. Khargonekar, I. R. Petersen, and K. Zhou, "Robust stabilization of uncertain linear systems: quadratic stabilizability and $H^{\infty}$ control theory," IEEE Transactions on Automatic Control, vol. 35, no. 3, pp. 356-361, 1990.

[7] M. Maki and K. Hagino, "Robust control with adaptation mechanism for improving transient behaviour," International Journal of Control, vol. 72, no. 13, pp. 1218-1226, 1999.

[8] H. Oya and K. Hagino, "Robust control with adaptive compensation input for linear uncertain systems," IEICE Transactions on Fundamentals of Electronics, Communications and Computer Sciences, vol. E86-A, no. 6, pp. 1517-1524, 2003.

[9] H. Oya and Y. Uehara, "Synthesis of variable gain controllers based on LQ optimal control for a class of uncertain linear systems," in Proceedings of the UKACC International Conference on Control (CONTROL '12), pp. 87-91, Cardiff, UK, September 2012.

[10] D. D. Šiljak, Decentralized Control of Complex Systems, vol. 184 of Mathematics in Science and Engineering, Academic Press, Boston, Mass, USA, 1991.

[11] C. Hua, X. Guan, and P. Shi, "Decentralized robust model reference adaptive control for interconnected time-delay systems," Journal of Computational and Applied Mathematics, vol. 193, no. 2, pp. 383-396, 2006.

[12] D. Xu, B. Jiang, H. Liu, and P. Shi, "Decentralized asymptotic fault tolerant control of near space vehicle with high order actuator dynamics," Journal of the Franklin Institute, vol. 350, no. 9, pp. 2519-2534, 2013. 
[13] C. J. Mao and W.-S. Lin, "Decentralized control of interconnected systems with unmodelled nonlinearity and interaction," Automatica, vol. 26, no. 2, pp. 263-268, 1990.

[14] C. J. Mao and J.-H. Yang, "Decentralized output tracking for linear uncertain interconnected systems," Automatica, vol. 31, no. 1, pp. 151-154, 1995.

[15] Z. Gong, "Decentralized robust control of uncertain interconnected systems with prescribed degree of exponential convergence," IEEE Transactions on Automatic Control, vol. 40, no. 4, pp. 704-707, 1995.

[16] H. Oya and K. Hagino, "Trajectory-based design of robust nonfragile controllers for a class of uncertain linear continuoustime systems," International Journal of Control, vol. 80, no. 12, pp. 1849-1862, 2007.

[17] H. Mukaidani, Y. Takato, Y. Tanaka, and K. Mizukami, "The guaranteed cost control for uncertain large-scale interconnected systems," in Proceedings of the Preprints of the 15th IFAC World Congress, Barcelona, Spain, 2002.

[18] H. Mukaidani, M. Kimoto, and T. Yamamoto, "Decentralized guaranteed cost control for discrete-time uncertain large-scale systems using fuzzy control," in Proceedings of the IEEE International Conference on Fuzzy Systems, pp. 635-641, Vancouver, Canada, July 2006.

[19] F. R. Gantmacher, The Theory of Matrices, vol. 1, Chelsea Publishing, Providence, RI, USA, 1960.

[20] S. Boyd, L. El Ghaoui, E. Feron, and V. Balakrishnan, Linear Matrix Inequalities in System and Control Theory, vol. 15 of SIAM Studies in Applied Mathematics, SIAM, Philadelphia, Pa, USA, 1994. 

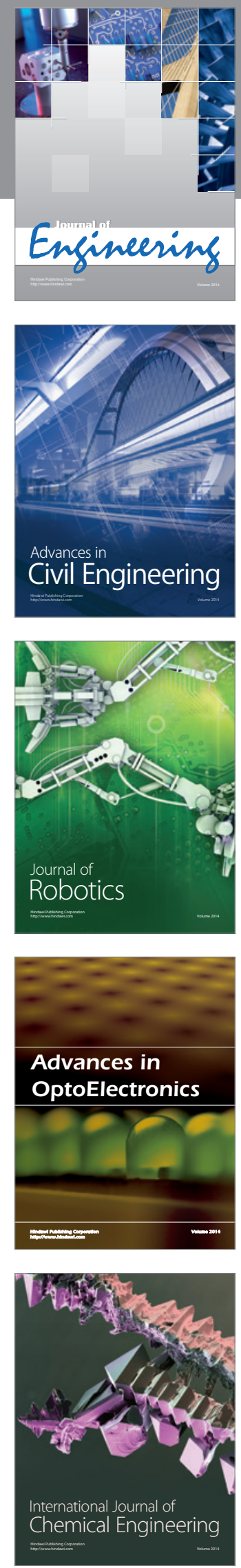

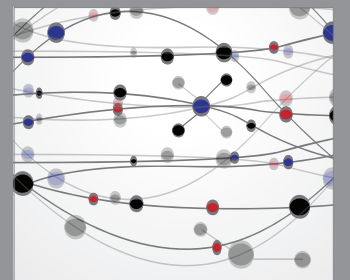

The Scientific World Journal
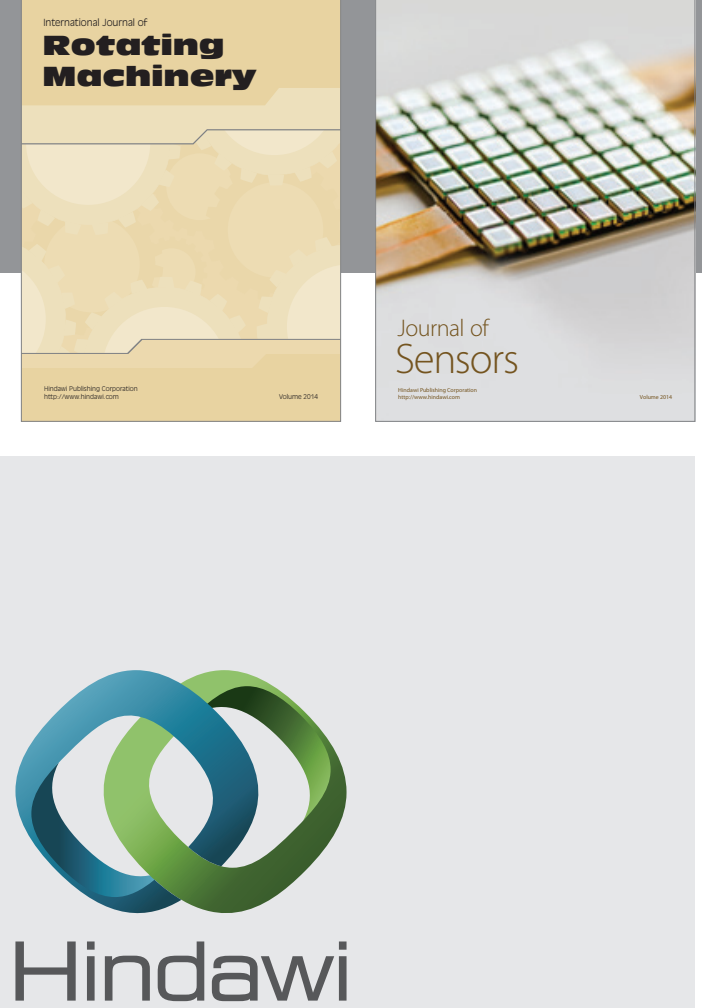

Submit your manuscripts at http://www.hindawi.com
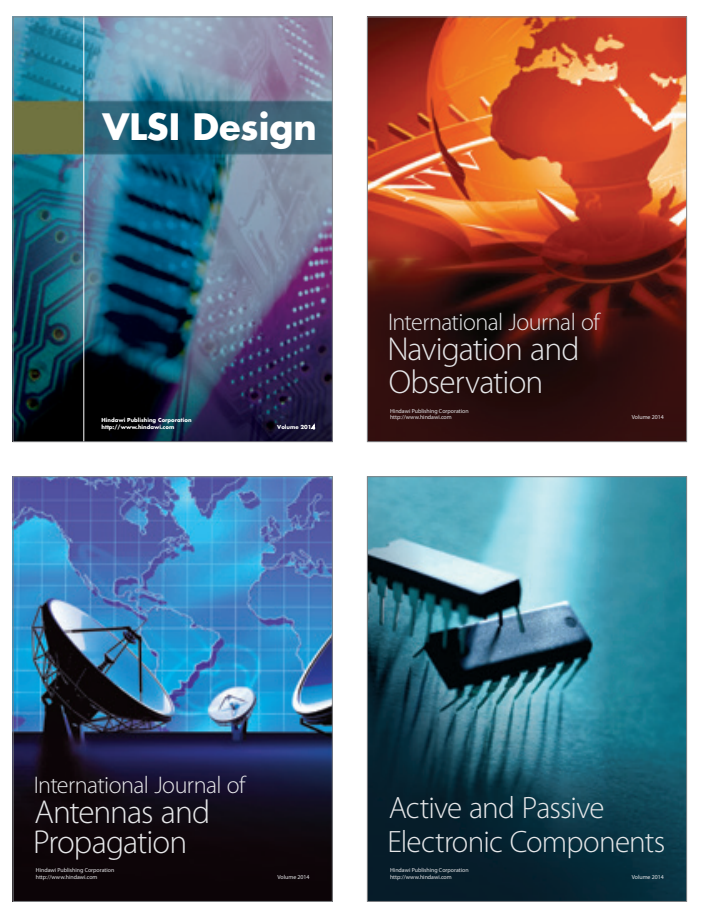
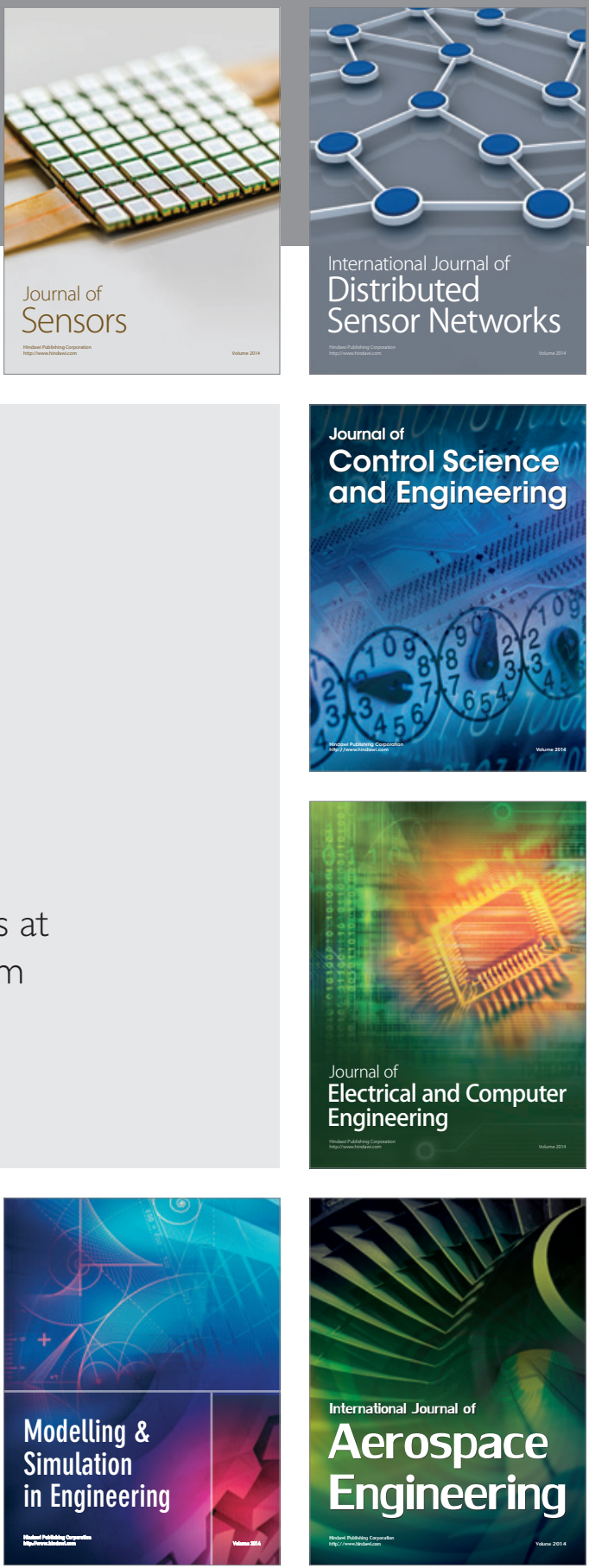

Journal of

Control Science

and Engineering
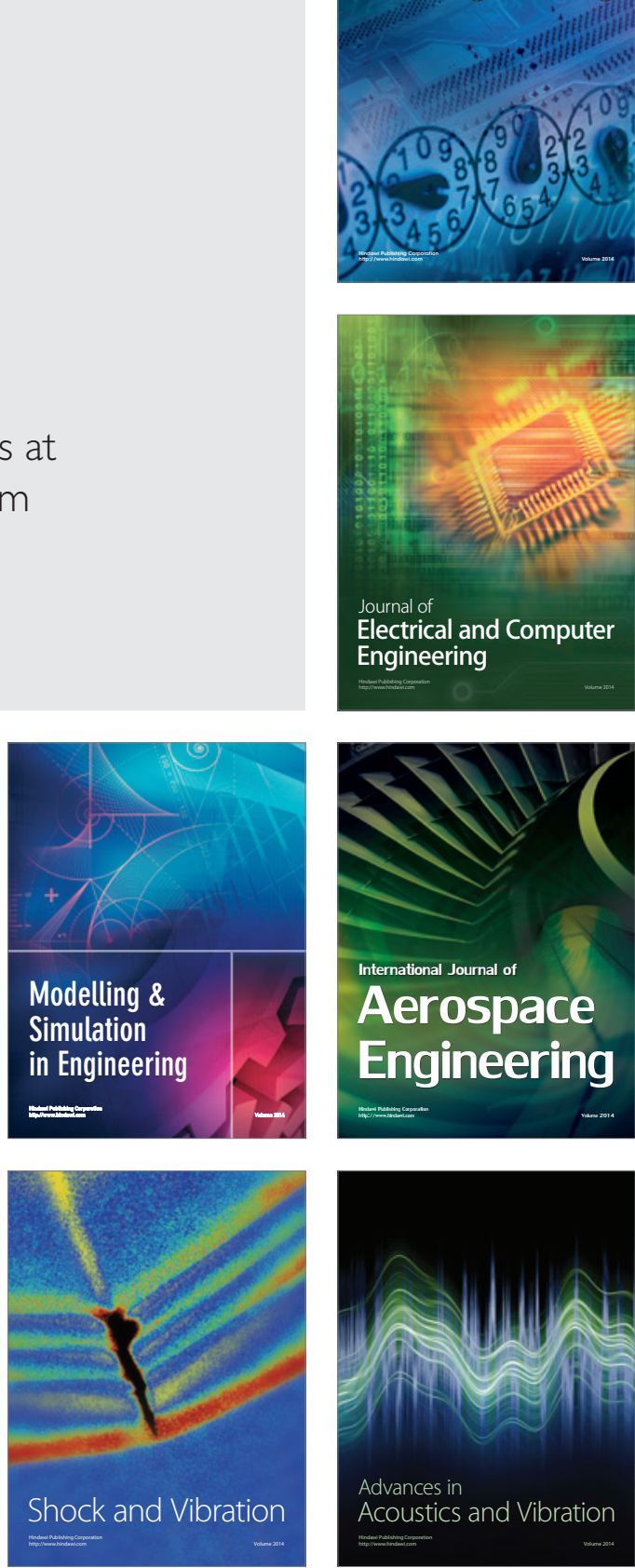